\title{
Surface Refractivity Gradient Data for Radio System Design
}

\author{
Steve J. Salamon ${ }^{* 1,3}$, Hedley J. Hansen ${ }^{2}$, and Derek Abbott ${ }^{1}$ \\ ${ }^{1}$ School of Electrical and Electronic Engineering, University of Adelaide, South Australia 5005, \\ stephen.salamon@adelaide.edu.au, derek.abbott@adelaide.edu.au
}

${ }^{2}$ RFT group CEWD, DSTO, PO Box 1500 Edinburgh, South Australia 5111, hedley.hansen@dsto.defence.gov.au

${ }^{3}$ Radio Transport, Telstra Corporation, 30 Pirie St Adelaide, South Australia 5000, steve.salamon@team.telstra.com

\begin{abstract}
Reliable information about the cumulative distribution of surface refractivity gradient is often required in the design of radio systems. Strong negative gradients, or super-refraction, may lead to interference between terrestrial stations, both terrestrial links and satellite earth stations. Predicted positive or sub-refractive gradients are taken into account in determining the minimum antenna heights, to ensure terrestrial links achieve their required availability. This paper reviews sources of refractivity gradient data, and considers how refractivity gradient at a point may relate to an effective value over a terrestrial path.
\end{abstract}

\section{Introduction}

The radio refractive index, or radio refractivity, can be estimated from the meteorological parameters of air temperature $T$, pressure $P$, and water vapour pressure $e$. Refractivity $N$, in "N-units", or parts-per-million by which the refractive index exceeds unity, is often estimated by [1]

$$
N=N_{\text {dry }}+N_{\text {wet }}=\frac{77.6}{T}\left(P+4810 \frac{e}{T}\right)
$$

where $P$ : atmospheric pressure $(\mathrm{hPa})$

$$
\begin{aligned}
& T \text { : absolute temperature }(\mathrm{K}) \\
& e \text { : water vapour pressure }(\mathrm{hPa}) .
\end{aligned}
$$

Although $T$ and $P$ can be measured directly, $e$ can be estimated from measured dew-point [1]. Instruments that collect these parameters are carried aloft by balloons. Many of these instruments, known as radiosondes, are released regularly, once, twice or even four times a day, generally at times close to 00 and 12 hours Co-ordinated Universal Time (UTC), and also 06 and 18 hours UTC in the case of stations with ascents every 6 hours.

Experimental measurements of these parameters on fixed towers are sometimes used to obtain surface refractivity gradient distributions [2,3], and measurements on towers in France have been used to develop an empirical model for gradient distribution as a function of median surface refractivity and median refractivity gradient [4].

Radiosonde data, with a sufficient number of ascents to provide reliable information in the tails of the gradient distribution, are only available from a limited number of stations - so empirical models to estimate the cumulative distribution of surface gradient from surface meteorological data have been developed. The Harvey model [5] predicts the most extreme value of positive refractivity gradient to be expected, while a recent model [6] predicts the refractivity gradient cumulative distribution from surface refractivity data. This paper presents a simplified version of the latter model. It predicts extreme values of the gradient distribution, and then predicts the distribution by interpolation.

Another source of surface gradient data is provided by numerical meteorological models, which implement a numerical solution to the meteorological conservation equations. World-wide models of this type can provide the parameters of (1) at a number of levels in the atmosphere, to produce digital maps of surface gradient distribution [1].

Considering that extreme gradient events may have limited geographic extent, especially in the case of subrefractive events, this paper describes techniques to estimate the sub-refractive tail of the distribution of mean surface gradient over a radio path of arbitrary length. 


\section{Radiosonde Data}

There are a very limited number of stations regularly providing data from ascents at 6 hourly intervals. These provide very good information about the local surface gradient distribution, as the ascents are spread throughout the day. Many more stations around the world provide radiosonde data at 12 hourly intervals, but caution is required in using data from stations with 24 hourly data, as gradient distributions can vary considerably depending on the local time of day that the ascents occur. Figure 1 illustrates this for a station in India. The data is from the NOAA/ESRL Radiosonde Database [7], processed as described in [6].

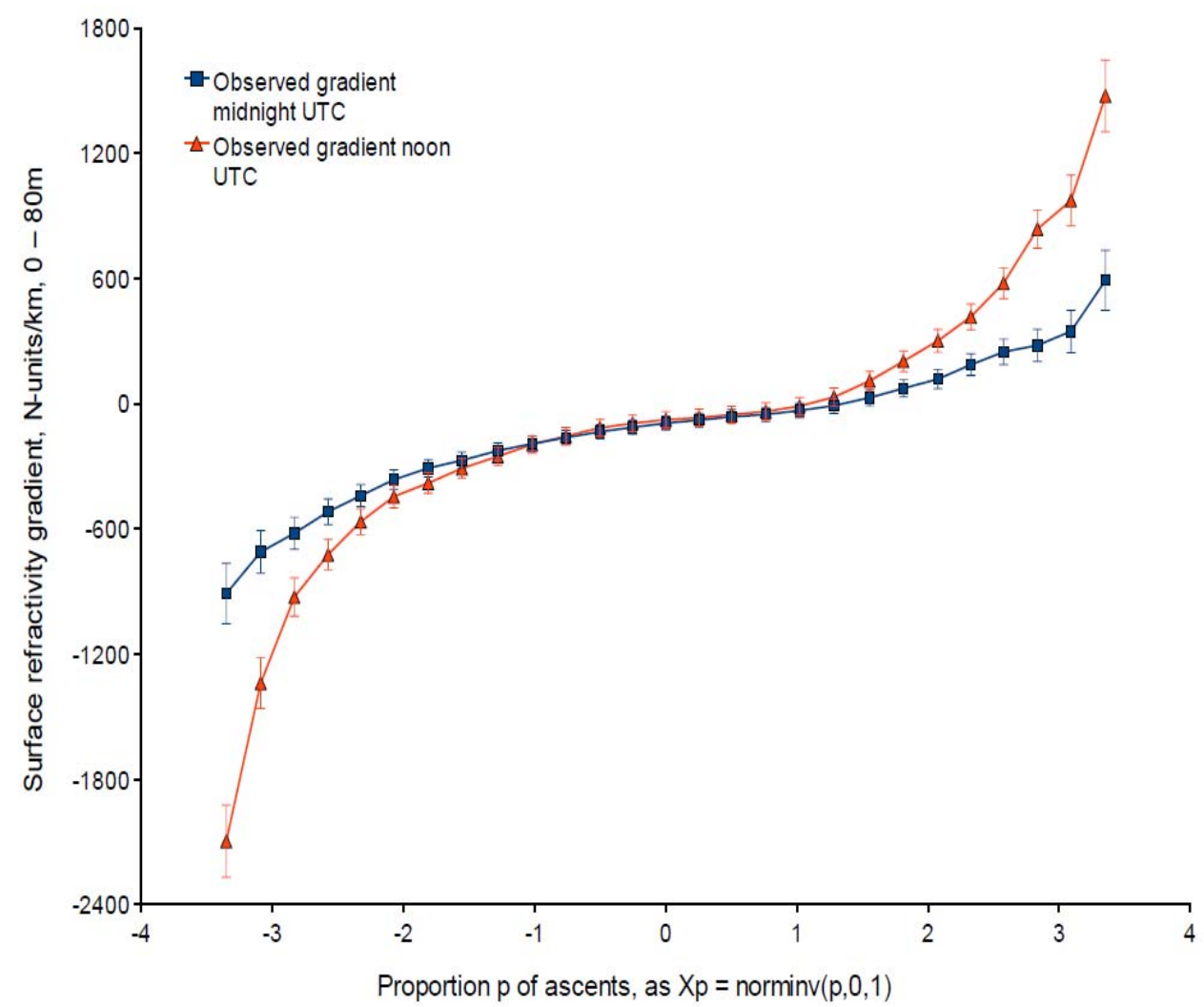

Figure 1. Radiosonde observed gradient distributions for Aurangabad, India, latitude 19.85 degrees, longitude 75.4 degrees, at around midnight UTC (early morning local time) and noon UTC (late afternoon local time).

\section{Gradient Prediction from Surface Data}

An empirical model has recently been developed [6], which predicts the surface gradient distribution from the distribution of a variable $\Delta N_{\mathrm{s}}$, referred to as "surface refractivity anomaly," which is the difference between surface refractivity $N_{\mathrm{s}}$ and its median value for that season of the year and hour of the day. A simpler model based on this work is obtained by taking only the $0.01 \%$ and $99.99 \%$ tail-points of the $\Delta N_{\mathrm{s}}$ distribution, $\Delta N_{\mathrm{s} 0.01}$ and $\Delta N_{\mathrm{s} 99.99}$, to predict the $0.01 \%$ and $99.99 \%$ tail-points of the $0-80 \mathrm{~m}$ surface gradient distribution, $G_{80}(0.01 \%)$ and $G_{80}(99.99 \%)$.

Linear regression analysis of data from 60 radiosonde stations provides the following model, firstly for the super-refractive tail-point, in units of $\mathrm{N}$-units per $\mathrm{km}$,

and the sub-refractive tail-point, in the same units,

$$
G_{80}(0.01 \%)=216-7.86\left(\Delta N_{\mathrm{s} 99.99}-\Delta N_{\mathrm{s} 0.01}\right),
$$

$$
G_{80}(99.99 \%)=-25+9.8 \max \left(-\Delta N_{\mathrm{s} 0.01}, \quad 0.75 \Delta N_{\mathrm{s} 99.99}\right) .
$$

We consider the distribution in terms of an inverse cumulative normal probability variable

$$
X_{\mathrm{p}}=\operatorname{norminv}(p, 0,1) \text {. }
$$


Denoting the values of $X_{\mathrm{p}}$ corresponding to the $0.01 \%$ and $99.99 \%$ tail points as $X_{0.01}$ and $X_{99.99}$, the complete cumulative distribution of refractivity gradient can be estimated by interpolation, using a modified sinh function:

$$
\begin{aligned}
& \begin{array}{r}
G_{80}\left(X_{\mathrm{p}}\right)=\left(G_{80}(99.99 \%)+1.7 C\right) \exp \left[A\left(X_{\mathrm{p}}-X_{99.99}\right)\right] \\
+\left(G_{80}(0.01 \%)-1.47 C\right) \exp \left[B\left(X_{0.01}-X_{\mathrm{p}}\right)\right]-2 C \tanh \left(X_{\mathrm{p}} / 4\right) \\
\quad+D\left(X_{\mathrm{p}} / X_{99.99}-1\right)\left(X_{\mathrm{p}} / X_{99.99}+1\right)
\end{array} \\
& \text { with } \quad A=\frac{8000}{5000+G_{80}(99.99 \%)} \quad \text { and } \quad B=\frac{4000-G_{80}(0.01 \%)}{8000},
\end{aligned}
$$

and $C$, which reduces the slope of the distribution in the region of the median, is given by

$$
C=-G_{80}(99.99 \%) A \exp \left(-A X_{99.99}\right)+G_{80}(0.01 \%) B \exp \left(B X_{0.01}\right) \text {. }
$$

If the median gradient $G_{80}(50 \%)$ is known, or can be predicted, then

$$
D=\left[G_{80}(99.99 \%)+1.7 C\right] \exp \left(-A X_{99.99}\right)+\left[G_{80}(0.01 \%)-1.47 C\right] \exp \left(B X_{0.01}\right)-G_{80}(50 \%)
$$

alternatively set $D=0$ if the median gradient is unknown. The value of the median gradient for the lowest 65 metres of the atmosphere, interpolated from the Rec ITU-R P.453 digital map [1], is used as an estimate of $G_{80}(50 \%)$ in $(8)$, for the testing of this model described below.

\section{Testing the Predicted Gradient Distributions}

Tables 1 and 2 below present error statistics for the ITU-R digital maps [1], the model of [6], and the revised version of that model in Section 3 above, when compared with the gradient distributions obtained by interpolating the radiosonde data [6]. Independent radiosonde data from 47 stations, not involved in the development of either of the new models, was used in these tests. Also the geographical distribution was different, with data from 33 Australian stations included in the 60 used to develop the models, but none included in the testing, while stations from China, Korea, Japan, Thailand and Indonesia were included in the testing but not in the development of the models.

\begin{tabular}{|c|c|c|c|c|c|c|c|c|c|}
\hline \multirow{2}{*}{$\begin{array}{l}\text { Model under test, mean } \\
\text { error compared to } \\
\text { radiosonde data }\end{array}$} & \multicolumn{9}{|c|}{ Percentage of ascents } \\
\hline & $0.01 \%$ & $0.1 \%$ & $1 \%$ & $10 \%$ & $50 \%$ & $90 \%$ & $99 \%$ & $99.9 \%$ & $99.99 \%$ \\
\hline $\begin{array}{c}\text { ITU-R P.453 digital } \\
\text { maps, 0-65 m [1] }\end{array}$ & - & - & -6 & -7 & +8 & -21 & -121 & - & - \\
\hline $\begin{array}{c}\text { Prediction from surface } \\
\text { data, same ascents [6] }\end{array}$ & -28 & -92 & -99 & -61 & -21 & +6 & -11 & -54 & -168 \\
\hline $\begin{array}{c}\text { Prediction from surface } \\
\text { data, same ascents, eq.(5) }\end{array}$ & -100 & -75 & -34 & -5 & +3 & -16 & -56 & -52 & -8 \\
\hline
\end{tabular}

Table 1. RMS error, in N-units/km, of empirical gradient models and ITU-R Rec. P.453 digital maps, compared with data from radiosondes for the same heights: $0-80 \mathrm{~m}$ for the models and $0-65 \mathrm{~m}$ for the maps.

\begin{tabular}{|c|c|c|c|c|c|c|c|c|c|}
\hline $\begin{array}{c}\text { Model under test, RMS } \\
\text { error compared to } \\
\text { radiosonde data }\end{array}$ & \multicolumn{8}{|c|}{ Percentage of ascents } \\
\cline { 2 - 11 } & $\mathbf{0 . 0 1 \%}$ & $\mathbf{0 . 1 \%}$ & $\mathbf{1 \%}$ & $\mathbf{1 0 \%}$ & $\mathbf{5 0 \%}$ & $\mathbf{9 0 \%}$ & $\mathbf{9 9 \%}$ & $\mathbf{9 9 . 9 \%}$ & $\mathbf{9 9 . 9 9 \%}$ \\
\hline $\begin{array}{c}\text { ITU-R P.453 digital } \\
\text { maps, 0-65 m [1] }\end{array}$ & - & - & 159 & 96 & 36 & 44 & 154 & - & - \\
\hline $\begin{array}{c}\text { Prediction from surface } \\
\text { data, same ascents [6] }\end{array}$ & 204 & 173 & 140 & 90 & 39 & 47 & 102 & 229 & 406 \\
\hline $\begin{array}{c}\text { Prediction from surface } \\
\text { data, same ascents, eq.(5) }\end{array}$ & 231 & 152 & 81 & 47 & 28 & 39 & 96 & 173 & 305 \\
\hline
\end{tabular}

Table 2. Mean error, in N-units/km, of empirical gradient models and ITU-R Rec. P.453 digital maps, compared with data from radiosondes for the same heights: 0-80 m for the models and 0-65 $\mathrm{m}$ for the maps. 


\section{Effective Sub-refractive Gradient for a Radio Path}

The surface gradient data from radiosondes, or gradient distribution prediction models, as described in [6] or in Section 3 above, estimate the distribution surface gradient at a point, but especially in the case of severe sub-refractive gradients, they may only affect part of a radio path at any point in time. An early treatment [8] of this provides an effective gradient for a path of arbitrary length, for $99.99 \%$ of the time, in a "continental temperate" climate. Converting the effective Earth radius factor to refractivity gradient in $\mathrm{N}$-units per $\mathrm{km}$, this curve is accurately replicated by

$$
G_{\text {path }}(99.99 \%)=-13+\frac{2670}{d} \text {, }
$$

where $d$ : path length, km.

Based on observed median depression radio fading events and meteorological measurements in the Julia Creek area of Queensland, Australia, a generally more severe model has been proposed [5], which can be expressed in terms of refractivity gradients $G_{\text {point }}$ and $G_{\text {path }}$, in N-units per km, as

$$
G_{\text {path }}=157\left[(\sqrt{39 / d}) /\left(0.17+\frac{94.2}{157+G_{\text {point }}}\right)-1\right] \text {. }
$$

Refractivity profiles during sub-refractive events can be complex [5]. Extreme surface gradients, as suggested by the tails of the curves in Figure 1, are likely to non-linear, with gradient varying considerably with height. Even in milder cases, increased gradient variation is normally seen at low heights, below 20 metres, as compared with greater heights $[2,3]$. The vertical refractivity profile is likely to vary considerably with distance along the path.

\section{Conclusion}

Reasonably accurate prediction of the cumulative distribution of surface refractivity gradient appears to be possible at locations with long-term automatic weather station data, using the models described above. At any instant in time, refractivity may vary in a complex way with height and distance along a radio path, so a uniform refractivity gradient for the whole path may not adequately describe refractive effects on field strength.

\section{References}

1. Recommendation ITU-R P.453-10, "The radio refractive index: its formula and refractivity data," Geneva, February 2012.

2. M. Grabner and V. Kvicera, "Statistics of lower atmosphere refractivity in Czechia," Proc. Asia-Pacific Microwave Conference, 4, 2005, DOI: 10.1109/APMC.2005.1606923.

3. G.S. Zhamsuyeva, "Variation of radiometeorological parameters in the lower atmosphere near Lake Baikal," Proc. URSI Climpara '98, Ottawa April 1998, pp. 116-117.

4. L. Blanchard and H. Szun, "Results of measurements of refractivity on towers in France; improvement of the ITU Rec P.453-6 cumulative distribution model of refractivity gradient,” Proc. URSI Climpara '98, Ottawa, April 1998, pp. $112-115$.

5. R. A. Harvey, "A subrefractive fading model for microwave paths using surface synoptic meteorological data," IEEE Trans. Antennas and Propagation, AP-35, no. 7, July 1987, pp. 832-844.

6. S. J. Salamon, H. J. Hansen and D. Abbott, "Prediction of Surface Refractivity Gradient Distributions, from Weather Station Surface Data," European Conference on Antennas and Propagation, April 2014, in press.

7. National Oceanic and Atmospheric Administration, Earth System Research Laboratory, NOAA / ESRL Radiosonde Database, http://www.esrl.noaa/raobs/Welcome.cgi

8. L. Boithias and J. Battesti, "Protection contre les évanouissements sur les faisceaux Hertziens en visibilité," Annales des Telecommunications, 22, no. 9-10, 1967, pp. 230-242. 